\title{
POSITIVE PERIODIC SOLUTIONS OF \\ NONAUTONOMOUS FUNCTIONAL DIFFERENTIAL \\ EQUATIONS DEPENDING ON A PARAMETER
}

GUANG ZHANG AND SUI SUN CHENG

Received 5 November 2001

This article investigates the existence of positive periodic solutions for a firstorder functional differential equations of the form

$$
y^{\prime}(t)=-a(t) y(t)+\lambda h(t) f(y(t-\tau(t)))
$$

where $a=a(t), h=h(t)$, and $\tau=\tau(t)$ are continuous $T$-periodic functions. We will also assume that $T>0, \lambda>0, f=f(t)$ as well as $h=h(t)$ are positive, $\int_{0}^{T} a(t) d t>0$.

Functional differential equations with periodic delays appear in a number of ecological models. In particular, our equation can be interpreted as the standard Malthus population model $y^{\prime}=-a(t) y$ subject to perturbation with periodical delay. One important question is whether these equations can support positive periodic solutions. Such questions have been studied extensively by a number of authors (cf. $[1,2,3,4,6,7]$ and the references therein). In this paper, we are concerned with the existence and nonexistence of periodic solutions when the parameter $\lambda$ varies. For this purpose, we call a continuously differentiable and $T$-periodic function a periodic solution of (1) associated with $\lambda^{*}$ if it satisfies (1) when $\lambda=\lambda^{*}$. We show that there exists $\lambda^{*}>0$ such that (1) has at least one positive $T$-periodic solution for $\lambda \in\left(0, \lambda^{*}\right]$ and does not have any $T$-periodic positive solutions for $\lambda>\lambda^{*}$. Our technique is based on the well-known upper and lower solutions method (cf. [5]).

We proceed from (1) and obtain

$$
\left[y(t) \exp \left(\int_{0}^{t} a(s) d s\right)\right]^{\prime}=\lambda \exp \left(\int_{0}^{t} a(s) d s\right) h(t) f(y(t-\tau(t))) .
$$


After integration from $t$ to $t+T$, we obtain

$$
y(t)=\lambda \int_{t}^{t+T} G(t, s) h(s) f(y(s-\tau(s))) d s,
$$

where

$$
G(t, s)=\frac{\exp \left(\int_{t}^{s} a(u) d u\right)}{\exp \left(\int_{0}^{T} a(u) d u\right)-1} .
$$

Note that the denominator in $G(t, s)$ is not zero since we have assumed that $\int_{0}^{T} a(t) d t>0$.

It is not difficult to check that any $T$-periodic function $y(t)$ that satisfies (3) is also a $T$-periodic solution of (1). Note further that

$$
\begin{gathered}
0<N \equiv \min _{0 \leq s, t \leq T} G(t, s) \leq G(t, s) \leq \max _{0 \leq t, s \leq T} G(t, s) \equiv M, \quad t \leq s \leq t+T, \\
1 \geq \frac{G(t, s)}{\max _{0 \leq s, t \leq T} G(t, s)} \geq \frac{\min _{0 \leq s, t \leq T} G(t, s)}{\max _{0 \leq s, t \leq T} G(t, s)}=\frac{N}{M}>0 .
\end{gathered}
$$

Now let $X$ be the set of all real $T$-periodic continuous functions, endowed with the usual linear structure as well as the norm

$$
\|y\|=\sup _{0 \leq t \leq T}|y(t)|
$$

Then $X$ is a Banach space with cones

$$
\begin{aligned}
& \Phi=\{y(t) \in X: y(t) \geq 0\}, \\
& \Omega=\{y(t): y(t) \geq \sigma\|y\|, t \in R\},
\end{aligned}
$$

where $\sigma=N / M$.

Define a mapping $F: X \rightarrow X$ by

$$
(F y)(t)=\lambda \int_{t}^{t+T} G(t, s) h(s) f(y(s-\tau(s))) d s .
$$

Then it is easily seen that $F$ is completely continuous on bounded subsets of $\Omega$ and for $y \in \Phi$,

$$
(F y)(t) \leq \lambda M \int_{0}^{T} h(s) f(y(s-\tau(s))) d s
$$

so that

$$
(F y)(t) \geq \lambda N \int_{0}^{T} h(s) f(y(s-\tau(s))) d s \geq \sigma\|F y\|
$$


That is, $F \Phi$ is contained in $\Omega$.

Lemma 1. The mapping $F$ maps $\Phi$ into $\Omega$.

Lemma 2. Suppose that

$$
\lim _{u \rightarrow+\infty} \frac{f(u)}{u}=+\infty
$$

Let $I$ be a compact subset of $(0,+\infty)$. Then there exists a constant $b_{I}>0$ such that $\|u\|<b_{I}$ for all $\lambda \in I$ and all possible $T$-periodic positive solutions $u$ of (1) associated with $\lambda$.

Proof. Suppose to the contrary that there is a sequence $\left\{u_{n}\right\}$ of T-periodic positive solutions of (1) associated with $\left\{\lambda_{n}\right\}$ such that $\lambda_{n} \in I$ for all $n$ and $\left\|u_{n}\right\| \rightarrow$ $+\infty$ as $n \rightarrow \infty$. Since $u_{n} \in \Omega$,

$$
\min _{0 \leq t \leq T} u_{n}(t) \geq \sigma\left\|u_{n}\right\|
$$

By (11), we may choose $R_{f}>0$ such that $f(u) \geq \eta u$ for all $u \geq R_{f}$, and there exists $n_{0}$ such that $\sigma\left\|u_{n_{0}}\right\| \geq R_{f}$, where $\eta$ satisfies

$$
\sigma \eta N \lambda_{n_{0}} \int_{0}^{T} h(s) d s>1
$$

Thus, we have

$$
\begin{aligned}
\left\|u_{n_{0}}\right\| & \geq u_{n_{0}}(t)=\lambda_{n_{0}} \int_{t}^{t+T} G(t, s) h(s) f\left(u_{n_{0}}(s-\tau(s))\right) d s \\
& \geq \sigma \eta N \lambda_{n_{0}} \int_{0}^{T} h(s)\left\|u_{n_{0}}\right\| d s>\left\|u_{n_{0}}\right\| .
\end{aligned}
$$

This is a contradiction. The proof is complete.

Lemma 3. Suppose that

$$
f \text { is nondecreasing on }[0,+\infty) \text { and } f(0)>0 \text {. }
$$

Let (1) have a T-periodic positive solution $y(t)$ associated with $\bar{\lambda}>0$. Then (1) also has a positive $T$-periodic solution associated with $\lambda \in(0, \bar{\lambda})$.

Proof. In view of (3) and (15), we have

$$
\begin{aligned}
y(t) & =\bar{\lambda} \int_{t}^{t+T} G(t, s) h(s) f(y(s-\tau(s))) d s \\
& \geq \lambda \int_{t}^{t+T} G(t, s) h(s) f(y(s-\tau(s))) d s, \quad 0<\lambda \int_{t}^{t+T} G(t, s) h(s) f(0) d s .
\end{aligned}
$$


282 Bifurcation in functional differential equations

Let $\bar{y}_{0}(t)=y(t)$

$$
\bar{y}_{k+1}(t)=\lambda \int_{t}^{t+T} G(t, s) h(s) f\left(\bar{y}_{k}(s-\tau(s))\right) d s, \quad k=0,1,2, \ldots
$$

$\underline{y}_{0}(t)=0$, and

$$
\underline{y}_{k+1}(t)=\lambda \int_{t}^{t+T} G(t, s) h(s) f\left(\underline{y}_{k}(s-\tau(s))\right) d s, \quad k=0,1,2, \ldots
$$

Clearly, we have

$$
\bar{y}_{0}(t) \geq \bar{y}_{1}(t) \geq \cdots \geq \bar{y}_{k}(t) \geq \underline{y}_{k}(t) \geq \cdots \geq \underline{y}_{1}(t) \geq \underline{y}_{0}(t)
$$

If we now let $y(t)=\lim _{k \rightarrow \infty} \bar{y}_{k}(t)$, then $y(t)$ satisfies (3). Clearly, we have

$$
y(t) \geq \underline{y}_{1}(t)=\lambda \int_{t}^{t+T} G(t, s) h(s) f(0) d s>0 .
$$

This completes our proof.

Lemma 4. Suppose that (11) and (15) hold. Then there exists $\lambda_{*}>0$ such that (1) has a T-periodic positive solution.

Proof. Let

$$
\beta(t)=\int_{t}^{t+T} G(t, s) h(s) d s, \quad M_{f}=\max _{0 \leq t \leq T} f(\beta(t-\tau(t))), \quad \lambda_{*}=\frac{1}{M_{f}} .
$$

We have

$$
\begin{gathered}
\beta(t)=\int_{t}^{t+T} G(t, s) h(s) d s \geq \lambda_{*} \int_{t}^{t+T} G(t, s) h(s) f(\beta(s-\tau(s))) d s, \\
0<\lambda_{*} \int_{t}^{t+T} G(t, s) h(s) f(0) d s .
\end{gathered}
$$

Let $\bar{y}_{0}(t)=\beta(t)$,

$$
\bar{y}_{k+1}(t)=\lambda_{*} \int_{t}^{t+T} G(t, s) h(s) f\left(\bar{y}_{k}(s-\tau(s))\right) d s, \quad k=0,1,2, \ldots,
$$

$\underline{y}_{0}(t)=0$, and

$$
\underline{y}_{k+1}(t)=\lambda_{*} \int_{t}^{t+T} G(t, s) h(s) f\left(\underline{y}_{k}(s-\tau(s))\right) d s, \quad k=0,1,2, \ldots
$$


Clearly, we have

$$
\bar{y}_{0}(t) \geq \bar{y}_{1}(t) \geq \cdots \geq \bar{y}_{k}(t) \geq \underline{y}_{k}(t) \geq \cdots \geq \underline{y}_{1}(t) \geq \underline{y}_{0}(t)
$$

If we now let $y(t)=\lim _{k \rightarrow \infty} \bar{y}_{k}(t)$, then $y(t)$ satisfies (3). Clearly, we have

$$
y(t) \geq \underline{y}_{1}(t)=\lambda_{*} \int_{t}^{t+T} G(t, s) h(s) f(0) d s>0 .
$$

The proof is complete.

Theorem 5. Suppose that (11) and (15) hold. Then there exists $\lambda^{*}>0$ such that (1) has at least one positive T-periodic solution for $\lambda \in\left(0, \lambda^{*}\right]$ and does not have any $T$-periodic positive solutions for $\lambda>\lambda^{*}$.

Proof. Suppose to the contrary that there is a sequence $\left\{u_{n}\right\}$ of T-periodic positive solutions of (1) associated with $\left\{\lambda_{n}\right\}$ such that $\lim _{n \rightarrow \infty} \lambda_{n}=\infty$. Then either we have $\left\|u_{n_{j}}\right\| \rightarrow+\infty$ as $j \rightarrow \infty$ or there is $\tilde{M}>0$ such that $\left\|u_{n}\right\| \leq \tilde{M}$. Assume the former case holds. Note that $u_{n} \in \Omega$ and thus

$$
\min _{0 \leq t \leq T} u_{n}(t) \geq \sigma\left\|u_{n}\right\|
$$

By (11), we may choose $R_{f}>0$ and $\eta_{1}>0$ such that $f(u) \geq \eta_{1} u$ when $\sigma u \geq$ $R_{f}$. On the other hand, there exist $\left\{t_{n}\right\} \subset[0, T]$ such that $u_{n_{j}}\left(t_{n_{j}}\right)=\left\|u_{n_{j}}\right\|$ and $u_{n_{j}}^{\prime}\left(t_{n_{j}}\right)=0$ by the periodicity of $\left\{u_{n_{j}}(t)\right\}$. In view of $(1)$, we have

$$
\begin{aligned}
a\left(t_{n_{j}}\right)\left\|u_{n_{j}}\right\| & =a\left(t_{n_{j}}\right) u\left(t_{n_{j}}\right)=\lambda_{n_{j}} h\left(t_{n_{j}}\right) f\left(u_{n_{j}}\left(t_{n_{j}}-\tau\left(t_{n_{j}}\right)\right)\right) \\
& \geq \lambda_{n_{j}} \eta_{1} \sigma h\left(t_{n_{j}}\right)\left\|u_{n_{j}}\right\|
\end{aligned}
$$

for all large $j$. That is, we have $\lambda_{n_{j}} \leq a\left(t_{n_{j}}\right) /\left(\eta_{1} \sigma h\left(t_{n_{j}}\right)\right)$. Note that $a(t) / h(t)$ is bounded. Thus, we obtain a contradiction.

Next, suppose that the latter case holds. In view of (15), there exists $\eta_{2}>0$ such that $f(0) \geq \eta_{2} \tilde{M}$. Then as above, we will obtain

$$
\begin{aligned}
a\left(t_{n}\right)\left\|u_{n}\right\| & =a\left(t_{n}\right) u\left(t_{n}\right)=\lambda_{n} h\left(t_{n}\right) f\left(u_{n}\left(t_{n}-\tau\left(t_{n}\right)\right)\right) \\
& \geq \lambda_{n} \eta_{2} h\left(t_{n}\right) \tilde{M} \geq \lambda_{n} \eta_{2} h\left(t_{n}\right)\left\|u_{n}\right\|
\end{aligned}
$$

for all $n$. A contradiction will again be reached. 
Thus, there exists $\lambda^{*}>0$ such that (1) has at least one positive $T$-periodic solution for $\lambda \in\left(0, \lambda^{*}\right)$ and no $T$-periodic positive solutions for $\lambda>\lambda^{*}$.

Finally, we assert that (1) has at least one $T$-periodic positive solution for $\lambda=\lambda^{*}$. Indeed, let $\left\{\lambda_{n}\right\}$ satisfy $0<\lambda_{1}<\cdots<\lambda_{k}<\lambda^{*}$ and $\lim _{k \rightarrow \infty} \lambda_{k}=\lambda^{*}$. Since $u_{n}(t)$ is $T$-periodic positive solution of (1) associated with $\lambda_{n}$ and Lemma 2 implies that the set $\left\{u_{n}(t)\right\}$ of solutions is uniformly bounded in $\Omega$, the sequence $\left\{u_{n}(t)\right\}$ has a subsequence converging to $u(t) \in \Omega$. We can now apply the Lebesgue convergence theorem to show that $u(t)$ is a $T$-periodic positive solution of (1) associated with $\lambda=\lambda^{*}$. The proof is complete.

Example 6. Consider the equation

$$
x^{\prime}(t)+a(t) x(t)=\lambda h(t)\left\{x^{\gamma}(t-\tau(t))+1\right\}, \quad \gamma>1
$$

where $a, h$, and $\tau$ satisfy the same assumptions stated for (1). In view of Theorem 5 , there exists a $\lambda^{*}>0$ such that (30) has at least one $T$-periodic positive solution for $\lambda \in\left(0, \lambda^{*}\right]$ and no $T$-periodic positive solution for $\lambda>\lambda^{*}$.

Example 7. Consider the equation

$$
y^{\prime}(t)=-a y(t)+\lambda b\left(y^{2}(t)+\varepsilon\right)
$$

where $a, b, \varepsilon>0$. Note that the function $f(x)=\left(x^{2}+\varepsilon\right)$ satisfies (11) and (15) in Theorem 5. Therefore Theorem 5 may be applied. However, we may give a direct proof that, for $\lambda>a /(2 b \sqrt{\varepsilon})$, this equation cannot have any positive $2 \pi$-periodic solutions associated with $\lambda$. Indeed, assume to the contrary that $y(t)$ is such a solution. Then $y^{\prime}(\xi)=0$ for some $\xi \in[0,2 \pi]$. Hence

$$
-a y(\xi)+\lambda b y^{2}(\xi)+\lambda b \varepsilon=0
$$

However, since the discriminant of the quadratic equation

$$
\lambda b x^{2}-a x+\lambda b \varepsilon=0
$$

satisfies

$$
a^{2}-4 \lambda^{2} b^{2} \varepsilon<0
$$

a contradiction is obtained. We remark that when $\varepsilon=0$, our equation reduces to the well-known logistic equation.

Similarly, we can consider the equation

$$
x^{\prime}(t)=a(t) x(t)-\lambda h(t) f(x(t-\tau(t))),
$$


where $a=a(t), h=h(t)$, and $f=f(t)$ satisfy the same assumptions stated for (1). By (35), we have

$$
x(t)=\int_{t}^{t+T} H(t, s) h(s) f(x(s-\tau(s))) d s
$$

where

$$
H(t, s)=\frac{\exp \left(-\int_{t}^{s} a(u) d u\right)}{1-\exp \left(-\int_{0}^{T} a(u) d u\right)}=\frac{\exp \left(\int_{s}^{t+T} a(u) d u\right)}{\exp \left(\int_{0}^{T} a(u) d u-1\right)}
$$

which satisfies

$$
M \geq H(t, s) \geq N, \quad t \leq s \leq t+T,
$$

for some $M$ and $N>0$, and $\sigma=N / M \leq 1$.

Theorem 8. Suppose that (11) and (15) hold. Then there exists $\lambda^{*}>0$ such that (35) has at least one positive T-periodic solution for $\lambda \in\left(0, \lambda^{*}\right]$ and no T-periodic positive solution for $\lambda>\lambda^{*}$.

\section{Acknowledgment}

This work was supported by the Natural Science Foundation of Shanxi Province and Yanbei Normal College. Part of this work was done during the first author's visit to the Institute of Applied Mathematics, Academy of Mathematics and System Sciences, Chinese Academy of Sciences. The first author wishes to express his thanks to Professor Daomin Cao for his kind invitation and nice hospitality. We also thank the referee for his helpful criticisms.

\section{References}

[1] S. S. Cheng and G. Zhang, Existence of positive periodic solutions for non-autonomous functional differential equations, Electron. J. Differential Equations 2001 (2001), no. $59,1-8$.

[2] M. Fan and K. Wang, Optimal harvesting policy for single population with periodic coefficients, Math. Biosci. 152 (1998), no. 2, 165-177.

[3] Uniform ultimate boundedness and periodic solutions of functionaldifferential equations with infinite delay, J. Systems Sci. Math. Sci. 19 (1999), no. 3, 323-327 (Chinese).

[4] D. Q. Jiang and J. J. Wei, Existence of positive periodic solutions for nonautonomous delay differential equations, Chinese Ann. Math. Ser. A 20 (1999), no. 6, 715-720 (Chinese).

[5] Y.-H. Lee, Multiplicity of positive radial solutions for multiparameter semilinear elliptic systems on an annulus, J. Differential Equations 174 (2001), no. 2, 420-441. 
286 Bifurcation in functional differential equations

[6] Y. K. Li, Existence and global attractivity of positive periodic solutions for a class of delay differential equations, Science in China Series A 28 (1998), no. 2, 108-118 (Chinese).

[7] S. N. Zhang, Periodicity in functional-differential equations, Ann. Differential Equations 12 (1996), no. 2, 252-257.

Guang Zhang: Department of Mathematics, Yanbei Normal College and Datong College, Datong, Shanxi 037000, China

E-mail address: dtgzhang@yahoo.com.cn

Sui Sun Cheng: Department of Mathematics, Tsing Hua University, Hsinchu, TAIWAN 30043, TaIWAN

E-mail address: sscheng@math.nthu.edu.tw 


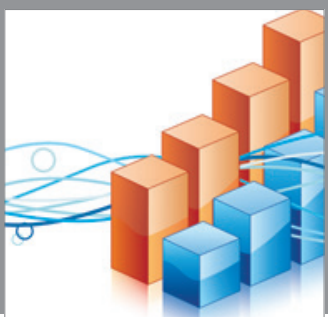

Advances in

Operations Research



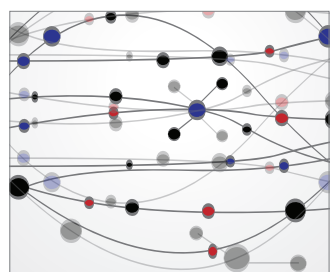

\section{The Scientific} World Journal
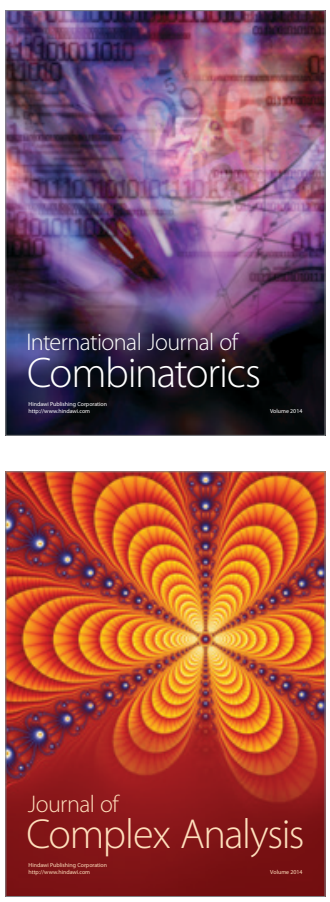

International Journal of

Mathematics and

Mathematical

Sciences


Journal of

Applied Mathematics
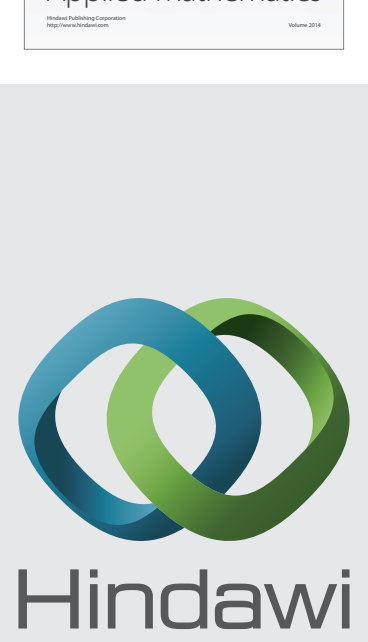

Submit your manuscripts at http://www.hindawi.com
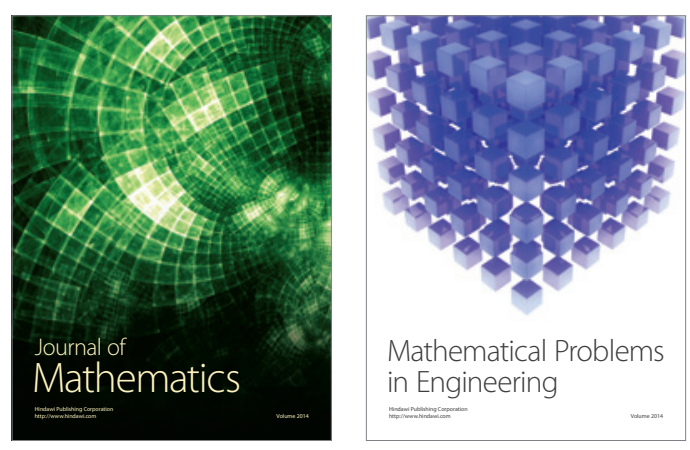

Mathematical Problems in Engineering


Journal of

Function Spaces
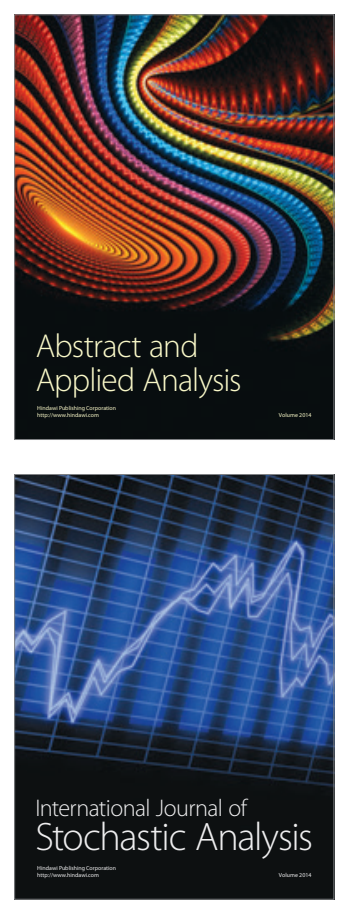



ournal of

Probability and Statistics

Promensencen
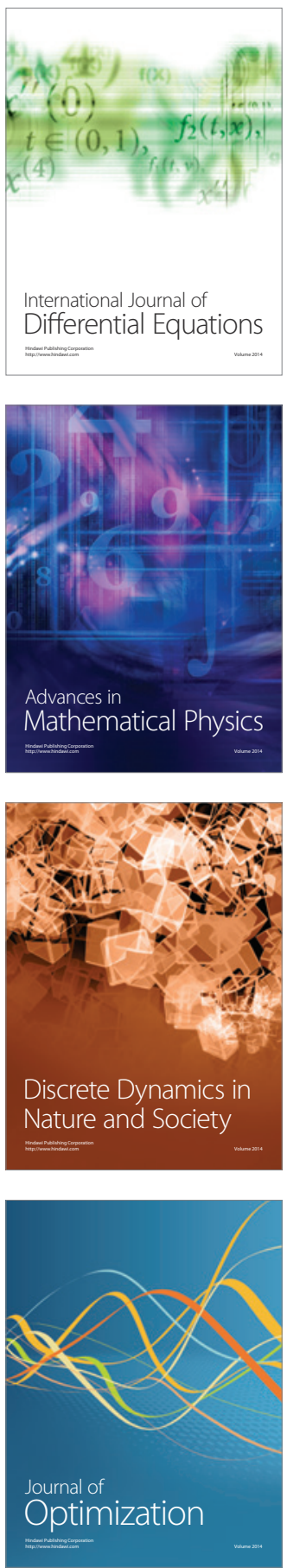